This item was submitted to Loughborough's Research Repository by the author.

Items in Figshare are protected by copyright, with all rights reserved, unless otherwise indicated.

\title{
Aerosol nucleation and growth in a turbulent jet using the Stochastic Fields
} method

PLEASE CITE THE PUBLISHED VERSION

http://dx.doi.org/10.1016/j.ces.2008.05.012

PUBLISHER

(C) Elsevier Ltd.

VERSION

AM (Accepted Manuscript)

LICENCE

CC BY-NC-ND 4.0

REPOSITORY RECORD

Garmory, Andrew, and E. Mastorakos. 2012. "Aerosol Nucleation and Growth in a Turbulent Jet Using the Stochastic Fields Method”. figshare. https://hdl.handle.net/2134/11117. 
This item was submitted to Loughborough's Institutional Repository (https://dspace.lboro.ac.uk/) by the author and is made available under the following Creative Commons Licence conditions.

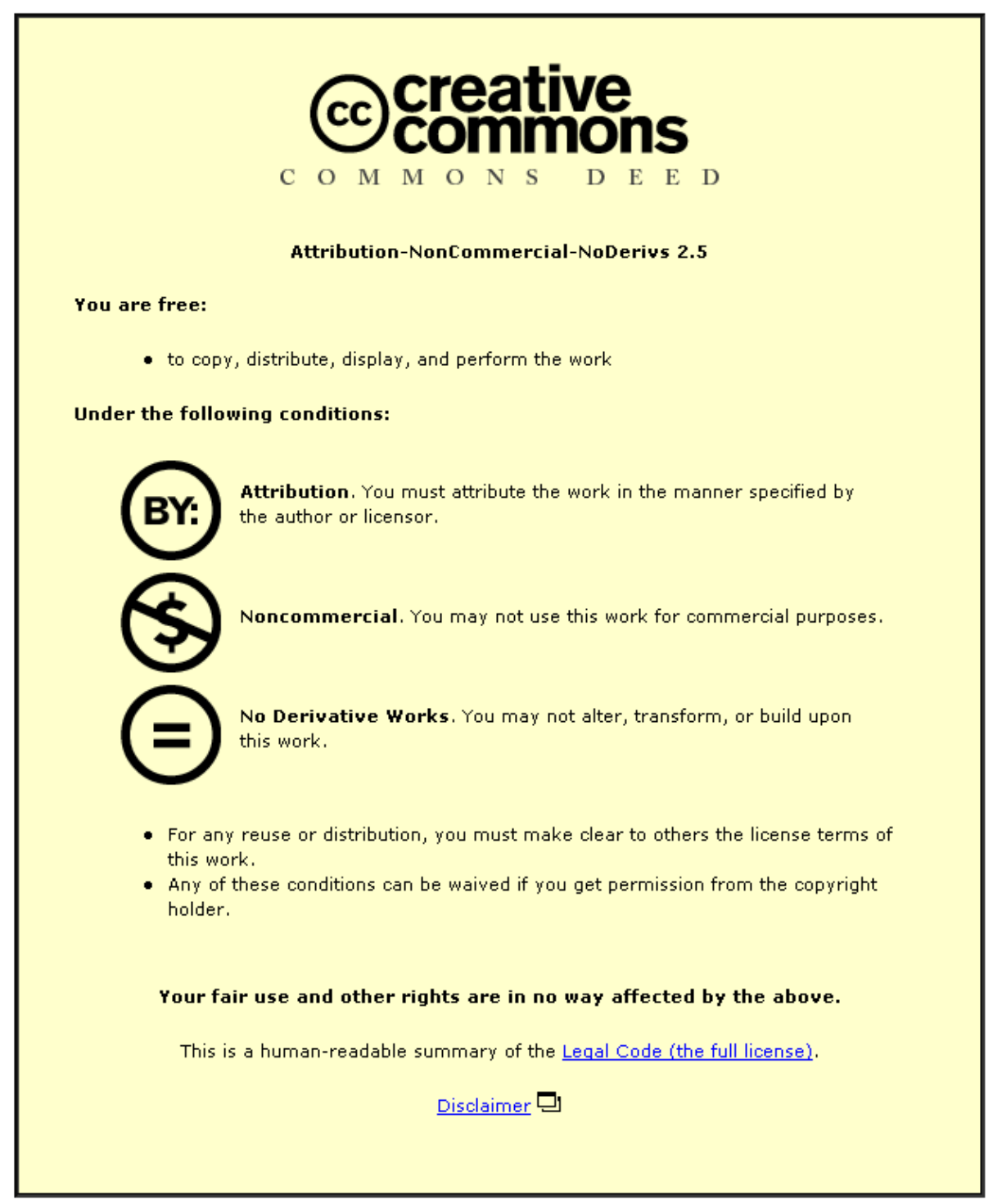

For the full text of this licence, please go to: http://creativecommons.org/licenses/by-nc-nd/2.5/ 


\title{
Aerosol Nucleation and Growth in a Turbulent Jet Using the Stochastic Fields Method
}

\author{
A. Garmory *and E. Mastorakos \\ Hopkinson Laboratory, Department of Engineering, University of Cambridge, UK
}

\begin{abstract}
The Stochastic Fields transported PDF method for turbulent reacting flows has been used to model the nucleation and growth of Dibutyl Phthalate particles in a hot, turbulent jet in a colder background for which experimental data is available. The aerosol population is modelled using an assumed log-normal size distribution. It has been found that neglecting the effect of turbulent fluctuations leads to the peak particle concentration being predicted too close to the jet and the concentration downstream underpredicted. However, this effect was small compared to that of adjusting modelled surface tension. Only by adjusting this was it possible to reproduce correctly the downstream evolution of particle number found in experiment. Particle mass mean diameter was significantly underpredicted at the centre of the jet, which may be due to the inability of log-normal size distribution to capture the distribution in detail. Taking account of turbulent fluctuations leads to increased mean particle size at the edge of the plume. The extent of this increase is strongly dependent on the choice of micromixing timescale.
\end{abstract}

Key words: Aerosol, Turbulence, Nucleation, Condensation, Monte Carlo, Stochastic Fields. 


\section{Introduction}

There are many situations where particles are produced in turbulent flows. These can be in industrial processes (Pratsinis, 1998) or in atmospheric flows where the particles, especially the smallest freshly nucleated ones, are known to be responsible for adverse health affects (Pope III, 2000; Seaton et al., 1995; Donaldson et al., 1998; Samet et al., 2000). The effect of turbulence on aerosol processes has been the subject of several recent studies (Lesniewski and Friedlander, 1995; Falk and Schaer, 2001; Moody and Collins, 2003; Rigopoulos, 2007). Wu and Menon (2001) investigated aerosol production in a turbulent jet engine wake. They employed the three moment system of Ford et al. (1996) together with the multiscale random mixing technique from their earlier paper (Menon and $\mathrm{Wu}, 1998)$ to investigate production of binary water and acid aerosols in aircraft plumes. They found that inclusion of random mixing events, as opposed to large scale turbulent entrainment alone, led to particle number density being increased by around 40\%. They attributed this increase to turbulent mixing bringing together $\mathrm{SO}_{3}$ and $\mathrm{H}_{2} \mathrm{O}$ to form $\mathrm{H}_{2} \mathrm{SO}_{4}$, which in turn leads to increased particle nucleation.

It has been found (Lesniewski and Friedlander, 1995; Wu and Menon, 2001; Falk and Schaer, 2001) that calculating nucleation, growth and coagulation terms as functions of mean quantitites can lead to significant errors compared to correctly including the effect of turbulence-induced fluctuations. Rigopoulos (2007) performs Reynolds averaging of the governing population balance equation for the evolution of an aerosol population, in order to examine the effects of turbulent fluctuations on these processes. It is shown that when this is done all three of nucleation, growth and coagulation have unclosed terms.

\footnotetext{
* Corresponding author.

Email address: ag310@cam.ac.uk (A. Garmory).
} 
While attempts have been made to model the effect of turbulence on coagulation via the collision frequency function (Saffman and Turner, 1956), this does not account for the correlation of fluctuations in number densities of particles of different sizes. These correlations were first noted by Scott (1967) and Warshaw (1967). The latter concluded that the correlations would have a greater effect in small volumes of air rather than in large volumes such as clouds. Friedlander (2000) states that these correlations require further study.

The homogeneous nucleation rate is a highly non-linear function of local vapour concentration and temperature. For instance a $10 \mathrm{~K}$ change in temperature can lead to changes in nucleation rate of several orders of magnitude. This non-linear dependence makes predicted nucleation rates extremely sensitive to the, usually empirical, expressions for surface tension, saturated vapour pressure and density used in the calculation. It also implies that attempting to calculate a nucleation rate for the mean population using only mean vapour concentration and temperature can lead to large errors. This is shown by Lesniewski and Friedlander (1995) who show that for nucleation at the edge of a turbulent jet a sharp ring of nucleation is predicted, if only mean quantities are used, but when turbulence is considered the nucleation region is spread out and the peak significantly lowered.

Hence it can be seen that turbulence has an affect on aerosol processes that would not be captured if only mean quantities were used in calculations. This non-linearity makes the use of Probability Density Function (PDF) methods, which capture the effect of fluctuations from the mean directly, highly attractive. Rigopoulos (2007) has obtained a transport equation for the joint particle number density - species PDF, i.e. $p\left(\phi_{1}, \ldots, \phi_{\alpha}, n_{1}, \ldots, n_{N}\right)$, where $\phi_{1}$ to $\phi_{\alpha}$ are the scalars and $n_{1}$ to $n_{N}$ are the number densities of particles in size bins from 1 to $N$. The equation is found to be similar to the scalar PDF transport 
equation (Fox, 2003) with nucleation, growth and coagulation appearing in closed form in the source term.

To accurately model an aerosol population a fine discretisation of the size distribution is required. This will lead to a high computational cost. The problem faced in using a full size distribution in a non-stochastic approach is multiplied by the number of realisations used in Monte-Carlo simulations typical of solution methods for PDF equation. There is, therefore, an advantage in using methods which assume a distribution shape can be characterised by a relatively small number of moments (see Section 2.2 and Pratsinis (1988); Frenklach (2002); Marchisio and Fox (2005) for more detail). If this is done then the object will be to find the joint scalar - size distribution moment PDF in the flow, e.g. $p\left(\phi_{v}, M_{0}, M_{1}, M_{2}, T\right)$ for a three moment system.

In this work an Eulerian Monte Carlo PDF method, the Stochastic Fields method, is employed to predict the nucleation and growth of Dibutyl Phthalate (DBP) particles in the early part of a turbulent jet. The objectives of this paper are (i) to implement the Stochastic Fields PDF method for aerosol nucleation and growth and (ii) to assess the importance of taking into account turbulent fluctuations on predictions of laboratory data (Lesniewski and Friedlander, 1998; Lesniewski, 1997), especially in comparison to sensitivity of predictions to the expression used for particle surface tension. The next section details the method used and its implementation here. Section 3 presents the results obtained, Section 4 contains a short discussion of these results and the paper closes with a summary of the conclusions drawn. 


\section{Formulation}

\subsection{Population Balance Equation}

The instantaneous local rate of change of the number concentration of particles in the size range $v$ to $v+d v$ is given by the population balance equation (Friedlander, 2000). This is a partial differential equation that models the simultaneous nucleation, growth, coagulation and transport of the aerosol population. It is expressed as:

$$
\begin{aligned}
\frac{\partial n}{\partial t} & +\nabla \cdot n \boldsymbol{u}+\frac{\partial(G n)}{\partial v} \\
& =\nabla \cdot D_{B} \nabla n+J\left(v^{*}\right) \delta\left(v-v^{*}\right)+\frac{1}{2} \int_{0}^{\infty} \beta\left(v-v^{\prime}, v^{\prime}\right) n\left(v-v^{\prime}, t\right) n\left(v^{\prime}, t\right) d v^{\prime} \\
& -n(v, t) \int_{0}^{\infty} \beta\left(v, v^{\prime}\right) n\left(v^{\prime}, t\right) d v^{\prime}
\end{aligned}
$$

The second term on the LHS of Eq. (1) represents advection by the carrier gas assuming low Stokes number, i.e. that the particles are sufficiently small to follow the flow with no slip. The third term on the LHS represents movement through the size distribution due to the growth of the particles, where $G(v)$ is the volume growth rate of particles of volume $v$ and will be a non-linear function of temperature and vapour concentration. The first term on the RHS describes diffusion of the particles due to Brownian motion. The second term on the RHS is the nucleation rate, $J$. Classical nucleation theory (Jacobson, 1999) gives:

$$
J=\frac{P_{v} N_{v} v_{m}}{k T}\left(\frac{2 \sigma}{\pi m}\right)^{1 / 2} \exp \left[-\frac{16 \pi \sigma^{3} m^{2}}{3(k T)^{3} \rho_{p}^{2}(\ln S)^{2}}\right]
$$

which is the rate of creation of new particles of a certain critical radius, $r_{c}$, 
given by:

$$
r_{c}=\frac{2 \sigma m}{\rho_{p} R^{*} T \ln S}
$$

where $T$ is the absolute temperature, $S$ is the saturation ratio, $\sigma$ is the surface tension of the droplet, $m$ is the mass of one molecule, $\rho_{p}$ is the density of the condensed liquid, $R^{*}$ is the universal gas constant, $P_{v}$ is the vapour partial pressure, $N_{v}$ is the vapour concentration, $v_{m}$ is the condensed molar volume and $k$ is Boltzmann's constant. The non-linearity of the nucleation rate can be seen in Eq. (2) and the errors associated with calculating this as a function of mean quantities only, as well as the strong dependence on $\sigma$, can be appreciated. The final two terms on the RHS of Eq. (1) describe respectively production and loss of particles of size $v$ due to coagulation. Solution of Eq. (1), which is an integro-differential equation, is difficult. Debry and Sportisse (2007a) and Debry and Sportisse (2007b) discuss this problem and present some new approaches.

\subsection{Aerosol Size Distribution}

To accurately model the evolution of an aerosol population a fine discretisation of the size distribution may be required, for which the computational cost may be prohibitive. If an assumed shape is used to represent the size distribution then it can be characterised by a number of moments (Pratsinis, 1988; Marchisio and Fox, 2005). A greater number of moments will mean that more information about the size distribution is contained in the calculation. The moments are defined as:

$$
M_{k}=\int_{0}^{\infty} v^{k} n(v) d v
$$


A governing equation for moment $M_{k}$ can be obtained by multiplying both sides of Eq. (1) by $v_{k}$ and integrating over all $v$. This leaves:

$$
\frac{\partial M_{k}}{\partial t}+\boldsymbol{u} \cdot \nabla M_{k}=\int_{0}^{\infty} v^{k} s d v
$$

where $\boldsymbol{u}$ is a turbulent velocity field and $s$ contains the nucleation, growth and coagulation terms from Eq. (1). When the integral on the RHS of Eq. (5) is performed (see Pratsinis (1988) for details) an expression is obtained for the source term of moment $k$ as a function of other moments. Some of these moments may be tracked as well, but others will not. For these unknown moments a closure is required. Frenklach (2002) shows that the logarithm of moments of a size distribution can be interpolated to find moments lying between those actually solved for. As with any interpolation scheme the accuracy of this can be increased by defining more points along the curve, i.e. solving for more moments.

Pratsinis (1988) solves for the first three moments of particle volume distribution and finds the required unknown moments by assuming that the distribution takes a log-normal shape. It can be shown that for any reasonable combination of the three known moments that the log-normal assumption produces a line in moment log-space that interpolates well between them. More accuracy could be obtained by solving for more moments along the curve, but here we employ the log-normal distribution because of its lower computational cost and complexity. The moments solved for are the zero, $M_{0}$, first, $M_{1}$, and second, $M_{2} . M_{0}$ is the total particle number concentration, while $M_{1}$ gives the total condensed volume per unit volume of gas. Dividing $M_{1}$ by $M_{0}$ gives mean particle volume which corresponds to volume mean diameter. If we assume that at a particular location all particles have uniform density this is equivalent to mass mean diameter. Results obtained by assuming that all 
particles have the same volume, i.e. monodisperse, are also included as a crude indication of the sensitivity to the assumed distribution shape.

\subsection{The Stochastic Fields Method}

The Stochastic Fields, or Field Monte Carlo, method is a transported PDF method developed for the simulation of turbulent reacting flows (Valiño, 1998; Sabel'nikov and Soulard, 2005b). Most Monte Carlo PDF methods employ the random advection through the flow of notional particles carrying the scalar values, the local PDF at a point can then be found by taking the ensemble of particles in the vicinity of that point (Pope, 1994). With the Stochastic Fields method a number of 'fields' extending across the whole spatial domain of the simulation are used. These fields contain values for each scalar at every node on an Eulerian grid. The evolution of each field takes place according to a governing stochastic partial differential equation (SPDE) derived from the scalar PDF transport equation. The Ito SPDE as derived by Valiño (1998), using the Interaction by Exchange with the Mean (IEM) closure for micromixing, is:

$$
\begin{aligned}
d \tau_{i}^{f} & =-U_{k} \frac{\partial \tau_{i}^{f}}{\partial x_{k}} d t+\frac{\partial}{\partial x_{k}}\left(K \frac{\partial \tau_{i}^{f}}{\partial x_{k}}\right) d t \\
& +\dot{w}\left(\tau_{1}^{f}, \tau_{2}^{f}, \ldots, \tau_{I}^{f}\right) d t+(2 K)^{1 / 2} \frac{\partial \tau_{i}^{f}}{\partial x_{k}} d W_{k}^{f}-\frac{\tau_{i}^{f}-\bar{\phi}_{i}}{T_{e d d y}} d t
\end{aligned}
$$

where $\tau_{i}^{f}$ is the value of scalar $i$ in field $f(f=1, \ldots, F), U_{k}$ is the mean velocity, $K$ is the combined molecular and turbulent diffusivity, $T_{e d d y}$ is a characteristic timescale of turbulent scalar mixing used in the IEM model and $\bar{\phi}_{i}$ is the local mean of scalar $i$. The PDF is then represented by $F$ stochastic fields $\tau^{f}$ which contain values for each scalar at each point throughout the flow. 
The Stochastic Fields method has to date been used to model combustion (Sabel'nikov and Soulard, 2005a; Mustata et al., 2006) and also gas phase atmospheric reacting flows (Garmory et al., 2006, 2007).

\subsection{Implementation of Stochastic Fields Method for Aerosol Processes}

The terms representing the effect of nucleation, growth and coagulation on the particle concentrations for each size bin appear in closed form in the source term of the PDF transport equation derived by Rigopoulos (2007), because in the equations used to model them they appear as production and loss terms that are functions purely of local conditions. Because of this they can be treated in the same way as other reacting scalars and appear in the source term as such. Terms are present in Rigopoulos's equation both for molecular diffusion of the ordinary scalar terms and also for diffusion of the particles. This particle diffusion will be due to Brownian motion and it is not at present clear how this should be modelled in the PDF approach. If this Brownian diffusion is dealt with in the same way as for the vapour phase and temperature scalars then the joint particle number density - species PDF can be treated in the same way as a standard joint scalar PDF equation, as was done in Rigopoulos (2007). Following the procedure of either Valiño (1998) or Sabel'nikov and Soulard (2005b) will allow the derivation of the Stochastic Fields equation in either Ito or Stratonovich form for the aerosol system.

If a moment method such as outlined in Pratsinis (1988) is to be used in a PDF simulation then the object is to solve for the evolution of $p\left(\phi_{v}, M_{0}, M_{1}, M_{2}, T\right)$ for a three moment system. This approach is followed by Falk and Schaer (2001), who simulate aggregation of silica particles in a precipitation reactor, and by Lindstedt and Louloudi (2005) in their transported PDF simulation 
of a jet flame with soot formation. In both cases a transported particle PDF approach is used, where each notional particle has a value for each scalar and also for each of a number of moments of the soot size distribution.

It can be seen that Eq. (5) models a quantity, $M_{k}$, which is dispersed by a turbulent velocity field and is produced and destroyed by source terms which are the integrated aerosol process term on the RHS. As will be seen below, these are treated in the models used for the RHS of Eq. (5) as being essentially functions of local vapour concentration, temperature and the other moments at the same location. As this is the case the system of scalars and moments can be treated in the same way as a system of reactive scalars and a modelled PDF transport equation can be derived with the aerosol processes in closed form in the source term. From this the Stochastic Fields equation can be derived in the usual way.

There is still an outstanding issue of the treatment of the micromixing term for the aerosols. Lindstedt and Louloudi (2005) apply Curl's mixing model to their notional particles. Falk and Schaer (2001) use the IEM model, with the mixing timescale set equal to the turbulent timescale taken from CFD, i.e. $T_{e d d y}=k / \epsilon$, for all scalars. If the IEM model is used and the timescale is assumed to be the same for all sizes of particle then integrating the IEM term over all particle sizes would yield the IEM term for the moments. In this paper we compare results assuming fluctuations in aerosol concentrations decay at the same rate as for vapour concentration with results neglecting any micromixing effect of the aerosol population.

The SF method coupled with an assumed size distribution should capture some of the effect of turbulence on particle size distribution and hence on aerosol processes. For example, if the first two moments are used, at a given 
point in a field a random combination of particle number and volume density will be found; thus the effect of correlations involving these two parameters is considered. However, in each field the distribution will conform to a similar shape, and the full effect of turbulent fluctuation on the shape of the distribution cannot be considered.

\subsection{Moment Source Terms}

In this work we use the three-moment log-normal system derived by Pratsinis (1988). Eq. (6) will be solved for five scalars in each field: vapour concentration, temperature and the first three moments of the size distribution. The source terms, $\dot{w}\left(\tau_{1}^{n}, \tau_{2}^{n}, \ldots, \tau_{5}^{n}\right)$, for the five scalars are:

$$
\begin{aligned}
\dot{w}_{\text {vap }} & =-\left(J v^{*}+C_{1}\right) / v_{m} \\
\dot{w}_{M_{0}} & =J-B_{1} \\
\dot{w}_{M_{1}} & =J v^{*}+C_{1} \\
\dot{w}_{M_{2}} & =J v^{* 2}+C_{2}+B_{2} \\
\dot{w}_{T} & =0
\end{aligned}
$$

where $v_{m}$ is the volume of a single molecule. $\dot{w}_{M_{0}}, \dot{w}_{M_{1}}$ and $\dot{w}_{M_{2}}$ correspond to the RHS of Eq. (5), while $\dot{w}_{v a p}$ is the source term for the vapour phase consistent with these. The critical volume $v^{*}$ is found from the critical radius in Eq. (3) and the nucleation rate is calculated using Eq. (2). The $B$ and $C$ terms account for coagulation and growth respectively. The coagulation terms, as given by Ford et al. (1996) working from Pratsinis (1988), for both the free molecule and continuum regimes are:

$$
\begin{aligned}
B_{1}^{F M} & =b_{0} \beta_{2}\left(M_{0}^{151 / 72} M_{1}^{-13 / 36} M_{2}^{19 / 72}\right. \\
& \left.+2 M_{0}^{131 / 72} M_{1}^{7 / 36} M_{2}^{-1 / 72}+M_{0}^{127 / 72} M_{1}^{11 / 36} M_{2}^{-5 / 72}\right) \\
B_{1}^{C} & =\beta_{4}\left(M_{0}^{2}+M_{0}^{19 / 9} M_{1}^{-2 / 9} M_{2}^{1 / 9}\right.
\end{aligned}
$$




$$
\begin{aligned}
& \left.+\beta_{5} \lambda\left(\frac{4 \pi}{3}\right)^{1 / 3}\left(M_{0}^{23 / 9} M_{1}^{-7 / 9} M_{2}^{2 / 9}+M_{0}^{25 / 9} M_{1}^{-11 / 9} M_{2}^{4 / 9}\right)\right) \\
B_{2}^{F M} & =2 b_{2} \beta_{2}\left(M_{0}^{19 / 72} M_{1}^{47 / 36} M_{2}^{31 / 72}\right. \\
& \left.+2 M_{0}^{-1 / 72} M_{1}^{67 / 36} M_{2}^{11 / 72}+M_{0}^{-5 / 72} M_{1}^{71 / 36} M_{2}^{7 / 72}\right) \\
B_{2}^{C} & =2 \beta_{4}\left(M_{1}^{2}+M_{0}^{1 / 9} M_{1}^{16 / 9} M_{2}^{1 / 9}\right. \\
& \left.+\beta_{5} \lambda\left(\frac{4 \pi}{3}\right)^{1 / 3}\left(M_{0}^{2 / 9} M_{1}^{17 / 9} M_{2}^{-1 / 9}+M_{0}^{4 / 9} M_{1}^{13 / 9} M_{2}^{1 / 9}\right)\right)
\end{aligned}
$$

where $\beta_{2}, \beta_{4}$ and $\beta_{5}$ are given by:

$$
\begin{aligned}
& \beta_{2}=\left(\frac{3}{4 \pi}\right)^{1 / 6}\left(\frac{6 k T}{\rho_{p}}\right)^{1 / 2} \\
& \beta_{4}=\left(\frac{2 k T}{3 \mu}\right) \\
& \beta_{5}=1.257
\end{aligned}
$$

and $b_{0}$ and $b_{2}$ are used in order to approximate the integral over all particle sizes and are given by

$$
\begin{aligned}
b_{0} & =0.633+0.092 a^{2}-0.022 a^{3} \\
b_{2} & =0.39+0.5 a-0.214 a^{2}+0.029 a^{3} \\
a & =\exp \left(\sqrt{\frac{1}{9} \ln \left(\frac{M_{0} M_{2}}{M_{1}^{2}}\right)}\right)
\end{aligned}
$$

$B_{1}$ and $B_{2}$ are found from the free molecule and continuum values by using a harmonic average. Kazakov and Frenklach (1998) found the use of harmonic averages to be adequate for most applications and that little is to be gained from the use of more complex methods.

The volume growth term in Eq. (1) is calculated according to Friedlander (2000):

$$
G=\left(48 \pi^{2}\right)^{1 / 3} D^{\prime} v^{1 / 3} v_{m} N_{s}(S-1)
$$


when integrated over all particle sizes according to Eq. (5) and using the log-normal assumption the moment growth terms $C_{1}$ and $C_{2}$ are found to be

$$
\begin{aligned}
& C_{1}=\left(48 \pi^{2}\right)^{1 / 3} v_{m} D^{\prime} N_{s}(S-1) M_{0}^{5 / 9} M_{1}^{5 / 9} M_{2}^{-1 / 9} \\
& C_{2}=2\left(48 \pi^{2}\right)^{1 / 3} v_{m} D^{\prime} N_{s}(S-1) M_{0}^{-1 / 9} M_{1}^{8 / 9} M_{2}^{2 / 9}
\end{aligned}
$$

$D^{\prime}=\omega D$ is the corrected molecular diffusion coefficent where $\omega$ is a correction factor to account for departure from continuum conditions and is a function of Knudsen number (Jacobson, 1999).

$$
\omega=\left[1+\left(\frac{1.33+0.71 K n^{-1}}{1+K n^{-1}}\right) K n\right]^{-1}
$$

$K n$ is calculated here as $\lambda / r_{m}$, where $r_{m}$ is the radius of a particle with mean volume and $\lambda$ is the mean free path in air. The molecular diffusion coefficient for DBP is calculated using (Jacobson, 1999)

$$
D_{D B P}=\frac{5}{16 A \rho_{a} d_{D B P}^{2}} \sqrt{\frac{R^{*} T M_{a}}{2 \pi}\left(\frac{M_{D B P}+M_{a}}{M_{D B P}}\right)}
$$

where $A$ is Avagadro's number, $\rho_{a}$ is the gas density, $M_{D B P}$ and $M_{a}$ are the molecular weights of Dibutyl Phthalate and air respectively and $d_{D B P}^{2}$ is the collision diameter of Dibutyl Phthalate estimated from the molecular volume. The constants and property values needed in the above expressions are given in Table 1.

\subsection{Model Problem}

Lesniewski and Friedlander (1998) and Lesniewski (1997) studied the homogeneous nucleation and growth of Dibutyl Phthalate (DBP) droplets in a turbulent axi-symmetric jet. DBP was chosen as it will condense at room temperature but will remain as vapour at temperature not far above this. This 
means that there is no need to cool the flow. The experiments were carried out in a $15 \mathrm{~cm}$ diameter glass chamber, and the jet nozzle diameter was either $0.235 \mathrm{~cm}$ or $0.375 \mathrm{~cm}$. The calculations presented in this paper use the 0.235 $\mathrm{cm}$ nozzle. The jet velocity was in the range $30-80 \mathrm{~m} \mathrm{~s}^{-2}$, in this paper a jet velocity of $51.5 \mathrm{~m} \mathrm{~s}^{-2}$ is investigated. A co-flow velocity of $0.18 \mathrm{~m} \mathrm{~s}^{-2}$ was used in the experiment to prevent recirculation. Measurements were restricted to the region where the jet could be considered a free jet, i.e. before the onset of plug-flow. For all experiments the co-flow was air at a temperature of $299 \mathrm{~K}$. The jet was at a temperature of $413 \mathrm{~K}$ and consisted of nitrogen with DBP vapour. The vapour concentration and temperature were chosen such that no nucleation would take place in the delivery system or on the nozzle and also so that measured number concentrations downstream would be in all cases less that $10^{4} \mathrm{~cm}^{-3}$. At this low number concentration the effect of coagulation can be expected to be negligible (Lesniewski and Friedlander, 1998).

\subsection{Numerical Methods}

In order to apply the Stochastic Fields method to this problem the threedimensional problem was transformed into a two-dimensional problem by taking a grid perpendicular to the jet axis and time marching Eq. (6) downstream from the nozzle exit. An axisymmetric formulation was not used as it is not clear what boundary conditions should be used on the axis for the stochastic fields (Sabel'nikov and Soulard, 2005b; Garmory, 2007). A fractional-step method was used in which the first step is to solve, for each field $n$ in turn, the advection, 'diffusion' and aerosol reaction terms employing the method of lines with the stiff ODE solver VODPK (Byrne, 1992).

$$
U_{1} \frac{\partial \tau_{i}^{f}}{\partial x_{1}}=-U_{k} \frac{\partial \tau_{i}^{f}}{\partial x_{k}}+\frac{\partial}{\partial x_{k}}\left(K \frac{\partial \tau_{i}^{f}}{\partial x_{k}}\right)+\dot{w}\left(\tau_{1}^{f}, \tau_{2}^{f}, \ldots, \tau_{5}^{f}\right)
$$


Here $\tau_{i}^{f}$ represents the value of the $i^{\text {th }}$ scalar in field $f$. The five scalars used for this problem are the concentration of DBP in the vapour phase $\left(\right.$ molec $\left.\mathrm{cm}^{-3}\right)$, temperature $(\mathrm{K})$ and the first three moments of the aerosol size distribution (particles per $\mathrm{cm}^{3}, \mathrm{~cm}^{3}$ of aerosol per $\mathrm{cm}^{3}$ gas and $\mathrm{cm}^{6}$ of aerosol per $\mathrm{cm}^{3}$ gas). $k=2,3$ and represents the two cross stream directions. $x_{1}$ is the axial distance from the nozzle exit and $U_{1}$ is the axial velocity.

The second step is to apply the random Wiener term to each scalar in each field using the Euler-Maruyama approximation. The local increment of the Wiener process in this case will be (Kloeden and Platen, 1999)

$$
\begin{aligned}
\Delta W_{k}^{f} & =\xi_{k}^{f}(\Delta t)^{1 / 2} \\
& =\xi_{k}^{f}\left(\frac{\Delta x_{1}}{U_{1}}\right)^{1 / 2}
\end{aligned}
$$

this makes the second fractional-step

$$
\tau_{i}^{f}(t+\Delta t)=\tau_{i}^{f}\left(t^{*}\right)+(2 K)^{1 / 2} \frac{\partial \tau_{i}^{n}\left(t^{*}\right)}{\partial x_{k}} \xi_{k}^{f}\left(\frac{\Delta x_{1}}{U_{1}}\right)^{1 / 2}
$$

where $\xi_{k}^{f}$ is a random number with zero mean and unity variance, chosen independently for each direction in each field. By calculating the integrand of Eq. (25) we ensure that it is independent of $\Delta W_{k}^{f}$ and that the Ito integral is obtained correctly (Gardiner, 2004). A limit is placed on the maximum size of the Wiener step to prevent unbounded scalars; this technique has been employed in previous work (Garmory et al., 2006, 2007) where it produced stable accurate solutions.

The third and final fractional step is to implement the micromixing term. This is done by using an analytical solution for the IEM term:

$$
\tau_{i}^{f}(t+\Delta t)=\bar{\phi}_{i}+\left(\tau_{i}^{f}\left(t^{*}\right)-\bar{\phi}_{i}\right) \exp \left(-\frac{1}{T_{e d d y}} \frac{\Delta x_{1}}{U_{1}}\right)
$$


For temperature and gas phase concentration the turbulent mixing timescale is made equal to the turbulent velocity timescale in line with previous studies using the IEM model (Jones and Kakhi, 1998; Garmory et al., 2006). For the aerosol moments it is as yet unclear what timescale should be used, or if the IEM model is particularly appropriate. Here we have adopted two methods; one is to assume that the mixing timescale is the same as for temperature and gas phase concentration, the second is to neglect any micromixing effect on the particles. By doing the latter the timescale $T_{\text {eddy }}$ for $M_{0}, M_{1}$ and $M_{2}$ is made effectively infinite and the micromixing term is dropped for these three. In order to assess the effect of turbulent fluctuations on predictions, calculations have also been carried out using a plain, non-stochastic, method. This is achieved by using only the first fractional step, Eq. (23), and neglecting Eqs. (25) \& (26). This gives a deterministic advection, diffusion reaction code with all terms evaluated at mean quantities.

Velocity and turbulence information in the form of $U_{k}, K$ and $T_{e d d y}$ were taken from a CFD simulation of the jet. The timescale $T_{e d d y}$ was calculated using $T_{\text {eddy }}=k / \epsilon$, where $k$ is the turbulent kinetic energy and $\epsilon$ is the turbulence dissipation rate. $K$ is found from from the turbulent viscosity found in the CFD solution using a turbulent Schmidt number of 0.7. FLUENT v6.2 was used to create the CFD solution. A 2D axisymmetric grid was used which extends 3 $\mathrm{cm}$ upstream of the end of the nozzle exit. This is equal to the length of tube used for the nozzle in the experiment and allows for the development of pipe flow in the simulation. The nozzle radius is $0.1175 \mathrm{~cm}$ and the grid extends $20 \mathrm{~cm}$ downstream of the nozzle and the maximum radius is $7.5 \mathrm{~cm}$. At the nozzle exit there are 19 cells across the pipe radius and the same resolution is initially used in the axial direction. Grid spacing in both radial and axial directions increases away from the nozzle. A total of 55,600 grid cells were used 
in the mesh. The solution was produced using the Reynolds stress model. The beginning of the nozzle tube and the co-flow were set to be mass-flow rate boundaries. With a temperature specified and the gauge pressure set to zero this is effectively the same as a velocity inlet. For the jet a mass flow rate of $1.378 \times 10^{-4} \mathrm{~kg} \mathrm{~s}^{-1}$ of nitrogen and a temperature of $413 \mathrm{~K}$ is specified, this corresponds to the $101 \mathrm{~min}^{-1}$ used in Lesniewski and Friedlander (1998). Turbulence boundary conditions of $k=0.001 \mathrm{~m}^{2} \mathrm{~s}^{-2}$ and $\epsilon=0.0001 \mathrm{~m}^{2} \mathrm{~s}^{-3}$ are used in the jet. The co-flow mass inlet extends from a radius of $1.35 \mathrm{~cm}$ to $7.5 \mathrm{~cm}$ (taken from scale drawing in Lesniewski (1997)) and a flow rate of $4.0833 \times 10^{-3} \mathrm{~kg} \mathrm{~s}^{-1}$ of air at a temperature of $299 \mathrm{~K}$. A turbulence intensity of $10 \%$ is used as measured in the experiment and a length scale of $1.5 \mathrm{~cm}$, equal to the diameter of the glass spheres placed upstream of the co-flow. Between the nozzle and the co-flow is a wall boundary condition representing the insulation around the nozzle.

A wall boundary is used at the edge of the domain and the outlet is specified as an outflow. The calculated axial velocity along the axis of the jet is shown in Fig. 1. Also shown is the velocity predicted by using an empirical expression from Tieszen et al. (1996) for axial velocity of a free jet with different density to the background:

$$
\frac{\bar{W}}{W_{0}}=11.8\left(\frac{\rho_{\text {jet }}}{\rho_{\text {back }}}\right)\left(\frac{r_{n o z}}{x_{1}}\right) \exp \left[-93.7\left(\frac{r}{x_{1}}\right)^{2}\right]
$$

$\bar{W}$ is the mean axial velocity, $W_{0}$ is the mean nozzle velocity equal here to $38.4 \mathrm{~m} \mathrm{~s}^{-1}$. There is very good agreement between the two solutions.

The SF equation was solved initially on a square $4 \mathrm{~cm}$ by $4 \mathrm{~cm}$ grid, centred on the jet axis, which was time-marched to $4.7 \mathrm{~cm}\left(\right.$ or $\left.x_{1} / d=20\right)$ downstream of the nozzle. At this point the grid was stretched to a $16 \mathrm{~cm}$ by $16 \mathrm{~cm}$ grid which was time-marched to 80 jet diameters downstream of the jet. The flow field 
data for the SF equation was provided using the axisymmetric CFD solution. At each SF step flow data at each grid point is found by calculating the axial and radial position at that point and interpolating linearly from this CFD section.

The grid spacing up to $x_{1} / d=20$ was $1.0 \times 10^{-2} \mathrm{~cm}$ up to $0.3 \mathrm{~cm}$ either side of the jet axis and $5.0 \times 10^{-2} \mathrm{~cm}$ outside this. Beyond $x_{1} / d=20$ the grid spacing was $4.0 \times 10^{-2} \mathrm{~cm}$ up to $0.3 \mathrm{~cm}$ either side of the jet axis and $2.0 \times 10^{-1} \mathrm{~cm}$ outside this. Results were also obtained using grid spacing double this (i.e. half resolution). The results, including variances, were found to be independent of the choice of grid and the finer grid was used unless stated. The axial distance step, $\Delta x_{1}$, in the time-marching scheme was varied between $1.0 \times 10^{-4} \mathrm{~cm}$ and $1.0 \times 10^{-2} \mathrm{~cm}$, it was again found that results were independent of this and therefore a value of $1.0 \times 10^{-3} \mathrm{~cm}$ was used. The micromixing term for vapour concentration and temperature was again carried out as a separate step to check that this caused no splitting errors. An initial condition of a top hat profile was used for vapour concentration and temperature with the nozzle value for those nodes in a $0.235 \mathrm{~cm}$ diameter circle and the background value for those outside. The three moments were set to low values across all nodes. The method outlined above, including limiting the size of the random term for the scalars, proved to be very robust even for these top hat profiles. A total of 30 fields were used for the SF calculations unless stated. The code took approximately 150 hours to run in parallel on three Pentium $43.0 \mathrm{GHz}$ CPU. 


\section{Results}

\subsection{Inert Mixing}

In order to test the parabolic Stochastic Fields scheme used in this work, results for the mean value of an inert scalar at various axial locations are compared with those obtained using the axisymmetric CFD solution used to produce the velocity and turbulence data. The inert scalar used is mixture fraction, i.e. a scalar which take a value of zero in the co-flow and one in the jet. The results are shown in Fig. 2. As will be the case throughout this chapter, the $2 \mathrm{D}$ SF solutions have been radially averaged to give a single radial profile at each axial location. This was done by taking the mean of all nodes whose radial distance to the centre line fell into the same size bin. The resolution of the size bins were equal to the grid spacing of the original grid. Fig. 2 shows good agreement between the two solutions, meaning that the SF solution is producing the correct mean mixing behaviour within the constraints of the velocity and turbulence data provided by the CFD solution.

\subsection{Axial and Radial Development of Aerosol Population}

\subsubsection{Particle Number Concentration}

To investigate the axial and radial development of the aerosol population, an initial temperature of $413 \mathrm{~K}$ and DBP vapour concentration of $360 \mathrm{ppm}$ was used. This corresponds to the conditions in trial 824 of Appendix D of Lesniewski (1997) for which centre-line measurements are available at several axial locations. Lesniewski (1997) and Okuyama et al. (1987) found that classical theory (Eq. (2)) under-predicts nucleation rates of DBP droplets by 
several orders of magnitude. This has also been observed here. Using initial vapour mole fractions in the range used in the experiment, 100 - $500 \mathrm{ppm}$, negligible nucleation rates were observed. This illustrates the difficulties of using classical theory to predict nucleation rates in complex situations.

Lesniewski (1997) suggests that the results can be reconciled to the experimental observation by using classical nucleation theory, but reducing the calculated surface tension by $10-15 \%$. Following this, the surface tension was initially reduced by $14 \%$. This corresponds to surface tension expression $\sigma(1)$ shown in Eq. (28) where surface tension is measured in $\mathrm{g} \mathrm{s}^{-2}$ and temperature is in Kelvin. It was found that by doing this it was possible to match experimental measurements of particle number at $x_{1} / d=20$ but not further downstream. This is shown in Fig. 3 where it can be seen that whether or not turbulent fluctuations are taken into account particle number is predicted to be increasing where, in fact, it should be decreasing. Still following the approach of Lesniewski (1997) in adjusting the value of surface tension in order to match experimental data, the dependence of $\sigma$ on temperature was investigated. By increasing the rate at which $\sigma$ falls with increasing temperature it was possible to find more nucleation in the hotter region close to the jet and less further downstream. Two such expressions are given in Eqs. (29) \& (30) and the results of using them are seen in Fig. 3.

$$
\begin{aligned}
& \sigma(1)=0.86(35.3-0.0863(T-273)) \\
& \sigma(2)=1.04(35.3-0.2(T-273)) \\
& \sigma(3)=1.10(35.3-0.22(T-273))
\end{aligned}
$$

The pre-multiplying factor is used to control the absolute values of nucleation rate.

Also shown in Fig. 3 is the effect of including turbulent fluctuations on the 
predicted centre-line particle number. For all three versions of surface tension, including turbulence via SF leads to shifting the peak concentration to further downstream. The concentration is initially reduced, but is increased further downstream. This agrees with the findings of Wu and Menon (2001) who found that turbulent mixing lead to an increase in production of $\mathrm{H}_{2} \mathrm{O} / \mathrm{H}_{2} \mathrm{SO}_{4}$ particles. However the difference due to turbulence is small in comparison with the effect of surface tension. Of the three expressions above, Eq. (29) gives predictions closest to the experimental data and this is used in all subsequent calculations.

Number concentration RMS along the centre-line is shown in Fig. 4. This is done for $\mathrm{SF}$ results using a mixing timescale for the aerosols equal to $T_{\text {eddy }}$ and also assuming an infinite mixing timescale. As expected assuming infinite micromixing time (i.e. no dissipation) leads to much higher RMS values which are of the order of the mean values. With a finite micromixing time the RMS values are reduced to about a half.

The choice of mixing timescale used has had very little effect on the predicted mean concentrations (although as expected the results found by neglecting micromixing are less smooth). This can be seen in Fig. 5 in which radial profiles of mean particle number density at four axial locations $\left(x_{1} / d=10\right.$, 20, $35 \& 65)$ are shown. The radial distances are normalised by the plume half radius, defined as the radius at which the mixture fraction is half that at the centre-line. The results contained in this figure were obtained using the Stochastic Fields method with both finite and infinite micromixing timescale for the aerosol moments. Results are also shown from a 'plain' advectiondiffusion-reaction simulation which uses only mean quantities and takes no account of segregation. It is not surprising that the two SF solutions are close as nucleation is a function of vapour concentration and temperature which 
have the same timescale in both solutions. If we compare the 'plain' and SF solutions it can be seen that in the early part of plume at $x_{1} / d=10$ the peak in particle density around the edge of the jet is significantly reduced in the SF solution compared to the 'plain' solution. Experimental data for these radial profiles is not available. By $x_{1} / d=20$ the peak concentration on the centre-line is reduced but is increased at the edge of the plume. Further downstream we see that the SF method predicts increased particle concentration right across the plume.

The effect of segregation can be further studied by considering the nucleation rate itself. The nucleation rate was calculated using both plain and SF methods. For the SF solution the nucleation rate was calculated in each field and then the mean was found at each grid node. This data was then radially averaged as before. Both axial and radial results of this are shown in Fig. 6. Only one set of results are used for the SF solution here as the results using both finite and infinite micromixing timescale for the aerosol moments are identical. Radial position has again been normalised by plume half-radius. The results show that while using mean values to calculate the nucleation rate leads to a sharp peak or 'flame' (Jenkins and Kennedy, 2000) of nucleation in both axial and radial directions. Whereas correctly taking account of turbulence, as with the SF method here, leads to a nucleation region that is wider and has a significantly lower peak.

The results obtained here for $x_{1} / d=10$ in Fig. 6(b) using the SF method show very good agreement with Figure 2 of Lesniewski (1997), which shows results found in Lesniewski and Friedlander's earlier paper (Lesniewski and Friedlander, 1995). In this they calculate reaction rate for one set of trial conditions, assuming unity Lewis number, in the shear layer using mean values and also by using measured PDF's for concentration and temperature from experimen- 
tal data. They showed that nucleation rate is a function of position in the shear layer and also that, while using mean values to calculate the nucleation rate leads to a sharp high peak, correctly taking account of turbulence leads to a nucleation region that is wider and has a significantly lower peak. This finding is reproduced here using the the Stochastic Fields method to calculate the PDF. Further downstream we found that total effect of having a smaller peak nucleation rate and a larger nucleation region leads to increased particle numbers downstream.

\subsubsection{Particle Size}

Fig. 7 shows the predicted mass mean diameter (mmd) at five axial locations $\left(x_{1} / d=20,35,50,65 \& 80\right)$ using four methods; (a) shows the results of ignoring turbulent fluctuations, (b) uses the SF method with mixing timescale equal to $T_{e d d y}=k / \epsilon$ and (c) uses the SF method with micromixing switched off. These first three methods assume a log-normal distribution while the fourth (d) assumes a monodisperse solution, i.e. locally all particles have the same volume (again with no micromixing). For the SF results the mmd is calculated in each field before the mean is taken. Also shown are the experimentally measured centre-line values. Experimental data for the radial profiles is not available.

For all four cases the centre-line mmd is significantly underpredicted at all five positions. For case (a) particle mmd is more or less uniform across the plume, whereas when the Stochastic Fields method is used with micromixing, case (b), there is a slight increase across the plume and in particular at the edge. When micromixing is switched off for the aerosol moments, case (c), a significant increase is seen at the edge of the plume with the mmd at the edge of the plume being two to three times that in the centre. The point at which 
the mmd starts to increase sharply moves towards the centre of the plume as the plume moves downstream. Case $(\mathrm{d})$, produced assuming a monodisperse distribution is very similar to that using the log-normal distribution.

Further detail about the predicted log-normal size distribution can be obtained by considering the geometric standard deviation, $S D_{g}$. This can be found from the three moments (Pratsinis, 1988) using

$$
\ln ^{2} S D_{g}=\frac{1}{9} \ln \left(\frac{M_{0} M_{2}}{M_{1}^{2}}\right)
$$

Radial profiles of this are shown in Fig. 8 at $x_{1} / d=20$ and 65 . When turbulent fluctuations are taken into account we see that the standard deviation is increased, i.e. the predicted distribution is wider. This increase is greater still when micromixing is switched off. Lesniewski and Friedlander (1998) reports measured geometric standard deviations of 1.7 which are notably higher than those predicted here.

Still using the log-normal distribution we can calculate a count mean diameter (cmd) from the mmd (Jacobson, 1999) which can be compared to experimental measurements. Radial profiles of this are shown in Fig. 9 at $x_{1} / d=20$ and 65. Here we find that at $x_{1} / d=20$ all methods used underpredict the cmd whilst at $x_{1} / d=65$ it is overpredicted, indicating that cmd values of the right magnitude are being found but that the growth rate is incorrectly predicted. Using the SF method with a finite mixing timescale leads to an increase in cmd across the width of the plume, particularly at the edge. With an infinite timescale the combined effect of the changed mmd and standard deviation seen in Figs. $7 \& 8$ leads to an increase in mean cmd at the edge of the plume but actually decreases it in the centre. Further discusion of these points is included in Section 4. 


\subsection{Effect of Varying Vapour Concentration}

Trial 819 of Appendix D of Lesniewski (1997) presents measurements of particle number concentration and mean diameter at a fixed point in the jet for varying jet vapour concentrations. This fixed point is the centre-line of the jet at $x_{1} / d=20$. Jet velocity and temperature were kept constant for all measurements. Our Stochastic Fields code has also been used to predict these measurements. Micromixing has been neglected for the aerosol moments in these calculations.

Fig. 10 shows the variation of particle concentration with jet vapour concentration. It can be seen that there is little difference between the plain and SF calculations, mostly due to the fact that a log-plot has been used. However inspection of the radial profiles for all results revealed that the same trend was seen as for Fig. 5. That is, that by including segregation the predicted peak number concentration occurs further downstream. These results are not included here for reasons of space. Results in the middle of the range show good agreement with the experimental results because the surface tension was tuned (as described in Section 3.2) to give agreement for this vapour concentration. However the predicted trend with vapour concentration is too steep for both plain and SF methods. Further tuning of the surface tension leads to higher or lower predicted values at all points but does not affect the gradient. Hence it appears that further study of the nucleation rate needs to be made in order to reconcile the predictions to the experimental data presented in Lesniewski (1997).

Fig. 11 shows predicted radial profiles of mmd at $x_{1} / d=20$ for four different initial vapour concentrations using the SF code with micromixing turned off. Also shown are experimentally measured points on the centre-line. We see 
that the profiles for all conditions collapse well onto each other with the sharp rise in mmd seen at around one half-radius. However once again, while the mmd at the edge of the plume is close to that measured on the centre-line, the predicted centre-line mmd is significantly underpredicted. Not shown are the corresponding results found without using the SF method. In line with Fig. 7 (a) these show mmd as approximately constant, and equal to the centre-line value predicted with SF, across the plume.

\section{Discussion}

Using the Stochastic Fields method to take account of turbulent fluctuations of vapour concentration and temperature led to predictions of nucleation rate which, compared to assuming mean quantities in the nucleation rate expression, had a lower peak rate but which were effective over a greater area. This agrees with Lesniewski and Friedlander (1995) where the nucleation rate was calculated using an experimentally measured PDF for vapour concentration and temperature. The changed nucleation rate due to the turbulence led to reduced particle number densities in the shear layer of the jet. Further downstream it was found that the cumulative effect of spreading the nucleation region over a larger area was to move the peak particle concentration further downstream and leads to higher particle concentrations over the width of the plume downstream.

However the sensitivity of our results to the inclusion of turbulent fluctuations is small compared to the sensitivity to the expression used for surface tension. This is not entirely surprising given that nucleation rate is a very strong function of surface tension and that estimating surface tension for very small particles is difficult (Jacobson, 1999). Predictions were reconciled with 
experimental results only by increasing by over a factor of two the rate at which $\sigma$ falls with increasing temperature. While the effect of turbulence described above was found to be the same for all versions of $\sigma$, it would appear that correct calculation of parameters such as surafce tension and saturation ratio are more important to the correct prediction of particle number.

Inclusion of turbulence was found to affect the predicted mass mean diameter. With no micromixing present for the aerosol moments a large increase in mmd was observed at the edge of the plume. Downstream this increase moved inwards towards the centre of the plume. With the inclusion of micromixing using a timescale $T_{e d d y}=k / \epsilon$ this increase was reduced and a smooth radial profile was observed rather than one with a sudden increase. The choice of timescale clearly has a large impact on the calculated mean particle sizes and therefore using a correct value will be crucial if accurate Monte Carlo PDF simulations of aerosol processes are to be made. Experimental data providing RMS, as well as mean, data on an aerosol population would allow the choice of timescale to be validated through RMS results.

Centre-line mmd's are underpredicted by all simulation methods here. Only by using the SF model with micromixing switched off was it possible to get mmd's as high as the measured centre-line values anywhere along the radial profile. However the predicted count mean diameters (cmd's) are of the same order as the measured data. Not predicting a big enough difference between cmd and mmd suggests that the assumed size-distribution is not adequate. An alternative, monodisperse, distribution was also used but this gave results very close to those from a log-normal distribution. It is likely to be the case that a wider particle distribution is needed to improve the predictions. This is supported by calculations of geometric standard deviation where values around 1.3 were found compared to measured values of 1.7 from Lesniewski and Friedlander 
(1998). For the higher initial vapour concentrations used in their experiments they also found size distributions with two or even three distinct peaks which certainly cannot be described by an assumed log-normal distribution. A wider distribution, or one with a separate peak at large radii, would give more particles at the higher extreme of the range. These large particles would increase, in particular, the mmd and would grow more rapidly according to Eq. (18). This could lead to more growth earlier in the plume rather than in the current results where cmd is wrongly seen to be still increasing past $x_{1} / d=20$.

An improvement in these results could be made by using a more accurate model for the distribution at a cost of CPU time and memory. This could be done by solving for a higher number of moments (Marchisio and Fox, 2005) or by using a full discretised size distribution (Rigopoulos, 2007). As well as providing a more accurate prediction of the size distribution the latter may also enable a greater insight into the effect of turbulence on aerosol processes via changes in the size distribution. The method used here allows this to a limited extent through fluctuations of the different moments of the distribution.

The experiment modelled was designed to avoid significant coagulation and the simulations presented here correctly predicted this. However, if coagulation was present then the importance of the shape of the size distribution and the effect of turbulence upon in would become even more important as this would be a function of the size distribution. It would be interesting to compare results using a joint-moment PDF method, as in this paper, with results found by calculating the joint PDF of the discretised size distribution (Rigopoulos, 2007) to assess the ability of the former method to model the effect of turbulence on coagulation. 


\section{Conclusions}

In this paper we have applied the Stochastic Fields transported PDF method to the nucleation and growth of Dibutyl Phthalate particles in a turbulent jet. This was done by solving for the first three moments of an assumed log-normal size distribution. It was found, in agreement with previous work (Lesniewski and Friedlander, 1995), that considering turbulent fluctuations of concentration and temperature led to a nucleation region that was wider but with a reduced peak nucleation rate. The result of this was that the peak particle number concentration was moved downstream. However this effect was small compared to that of adjusting the modelled surface tension. Also the predicted trend of centre-line particle number density with initial vapour concentration was found to be too steep compared to experimental results. It would appear that correct modelling of empirical expressions used for material properties is more important for predicting nucleation than the correct treatment of turbulent segregation in this case.

Centre-line mass mean diameters were significantly underpredicted while count mean diameters were predicted more closely. This, together with geometric standard deviations smaller than those reported by experiment, suggests that the log-normal distribution here is not capable of correctly representing the actual size distribution in this case, and in particular the presence of very large particles which would increase mmd without increasing cmd to the same degree. Future work using a greater numbver of moments or a discretised distribution would provide more accuracy.

The Stochastic Fields code showed that turbulent mixing of rapid growth conditions from the centre of the jet to the edge caused the mass mean diameter of the particles to be increased at the edge compared to calculations using only 
mean quantities. This increase was much greater when the mixing timescale was set to be infinite. This is equivalent to switching off the micromixing term representing the decay of turbulent fluctuations by diffusion. Hence choosing the correct timescale for micromixing will be critical when attempting to accurately model mean particle sizes.

\section{Acknowledgements}

This work has been funded by the Engineering and Physical Sciences Research Council.

\section{References}

G. D. Byrne. Pragmatic experiments with Krylov methods in the stiff ODE setting. In J. Cash and I. Gladwell, editors, Computational Ordinary Differential Equations. Oxford University Press, 1992.

E. Debry and B. Sportisse. Numerical solution of the general dynamic equation (GDE) for aerosols with two collocation methods. Applied Numerical Mathematics, 57:885-898, 2007a.

E. Debry and B. Sportisse. Solving aerosol coagulation with size-binning methods. Applied Numerical Mathematics, 57:1008-1020, $2007 \mathrm{~b}$.

K. Donaldson, X. Y. Li, and W. MacNee. Ultrafine (nanometer) particle mediated lung injury. Journal of Aerosol Science, 29:553-560, 1998.

L. Falk and E. Schaer. A PDF modelling of precipitation reactors. Chemical Engineering Science, 56:2445-2457, 2001.

I. J. Ford, G. D. Hayman, and R. D. Kingdon. Plume and aerosol modelling for the POLINAT project. In U. Schuman, editor, Pollution from Aircraft 
Emissions in the North Atlantic Flight Corridor (POLINAT), pages 258279. 1996.

R. O. Fox. Computational models for turbulent reacting flows. Cambridge University Press, Cambridge, 2003.

M. Frenklach. Method of moments with interpolative closure. Chemical Engineering Science, 57:2229-2239, 2002.

S. K. Friedlander. Smoke, Dust and Haze: Fundamentals of Aerosol Dynamics. Oxford University Press, Oxford, second edition, 2000.

C. W. Gardiner. Handbook of stochastic methods. Springer, Berlin, third edition, 2004.

A. Garmory. Micromixing effects in atmospheric reacting flows. PhD Thesis, University of Cambridge, 2007.

A. Garmory, E. S. Richardson, and E. Mastorakos. Micromixing effects in a reacting flow by the Stochastic Fields method. Atmospheric Environment, 40:1078-1091, 2006.

A. Garmory, R. E. Britter, and E. Mastorakos. Simulation of the evolution of aircraft exhaust plumes including detailed chemistry and segregation. To appear in Journal of Geophysical Research - Atmospheres, 2007.

M. Z. Jacobson. Fundamentals of atmospheric modeling. Cambridge University Press, Cambridge, 1999.

T. P. Jenkins and I. M. Kennedy. Measurements of aerosol product in an axisymmetric co-flow jet. Experiments in Fluids, 29:532-544, 2000.

W. P. Jones and M. Kakhi. PDF modeling of finite-rate chemistry effects in turbulent nonpremixed jet flames. Combustion and Flame, 115:210-229, 1998.

A. Kazakov and M. Frenklach. Dynamic modelling of soot particle coagulation and aggregation: implementation with the method of moments and application to high-pressure laminar premixed flames. Combustion and Flame, 
114:484-501, 1998 .

P. E. Kloeden and E. Platen. Numerical Solution of Stochastic Differential Equations. Springer, Berlin, corrected third printing edition, 1999.

T. K. Lesniewski. Particle Nucleation and Growth in Turbulent Jets. PhD Thesis, University of California, Los Angeles, 1997.

T. K. Lesniewski and S. K. Friedlander. The effect of turbulence on rates of particle formation by homogeneous nucleation. Aerosol Science and Technology, 23:174-182, 1995.

T. K. Lesniewski and S. K. Friedlander. Particle nucleation and growth in a free turbulent jet. Proceedings of the Royal Society, A, 454:2477-2504, 1998.

R. P. Lindstedt and S. A. Louloudi. Joint-scalar transported PDF modeling of soot formation and oxidation. Proceedings of the Combustion Institute, 30:775-783, 2005.

D. L. Marchisio and R. O. Fox. Solution of population balance equations using the direct quadrature method of moments. Journal of Aerosol Science, 36: 43-73, 2005.

S. Menon and J. Wu. Effects of micro- and macroscale turbulent mixing on the chemical processes in engine exhaust plumes. Journal of Applied Meteorology, 37:639-654, 1998.

E. G. Moody and L. R. Collins. Effect of mixing on the nucleation and growth of titania particles. Aerosol Science and Technology, 37:403-424, 2003.

R. Mustata, L. Valiño, C. Jiménez, W. P. Jones, and S. Bondi. A Probability Density Function Eulerian Monte Carlo field method for Large-eddy simulations. Application to a turbulent piloted methane/air diffusion flame (Sandia D). Combustion and Flame, 145:88-104, 2006.

K. Okuyama, Y. Kousaka, D. R. Warren, R. C. Flagan, and J. H. Seinfeld. Homogeneous nucleation by continuous mixing of high-temperature vapour by mixing with a cool gas. Aerosol Science and Technology, 6:15-27, 1987. 
S. B. Pope. Lagrangian PDF methods for turbulent flows. Annual Review of Fluid Mechanics, 26:23-63, 1994.

C. A. Pope III. Review: Epidemiological basis for particulate air pollution health standards. Aerosol Science and Technology, 32:4-14, 2000.

S. E. Pratsinis. Simultaneous nucleation, condensation and coagulation in aerosol reactors. Journal of Colloid and Interface Science, 124(2):416-427, 1988.

S. E. Pratsinis. Flame aerosol synthesis of ceramic powders. Progress in Energy and Combustion Science, 24:197-219, 1998.

S. Rigopoulos. PDF method for population balance in turbulent reactive flow. Chemical Engineering Science, 62:6865-6878, 2007.

V. Sabel'nikov and O. Soulard. Simulation of a turbulent premixed methane flame with an Eulerian Monte Carlo solver. In V. Dias and J. Vandooren, editors, Proceedings of the European Combustion Meeting, page E100, Louvain, 2005a. Belgian Section of The Combustion Institute.

V. Sabel'nikov and O. Soulard. Rapidly decorrelating velocity-field model as a tool for solving one-point Fokker-Planck equations for probability density functions of turbulent reactive scalars. Physical Review E, 72(1):Article No. 016301, 2005b.

P. G. Saffman and J. S. Turner. On the collision of drops in turbulent clouds. Journal of Fluid Mechanics, 1:16-30, 1956.

J. M. Samet, F. Dominici, F. C. Curriero, I. Coursac, and S. L. Zeger. Fine particulate air pollution and mortality in 20 U.S cities, 1987 - 1994. The New England Journal of Medicine, 343:1742-1749, 2000.

W. T. Scott. Poisson statistics in distributions of coalescing droplets. Journal of the Atmospheric Sciences, 24:221-225, 1967.

A. Seaton, W. MacNee, K. Donaldson, and D. Godden. Particulate air pollution and accute health effects. The Lancet, 345:176-176, 1995. 
S. R. Tieszen, D. W. Stamps, and T. J. O'Hern. A heuristic model of turbulent mixing applied to blowout of turbulent jet diffusion flames. Combustion and Flame, 106:442-466, 1996.

L. Valiño. A field Monte Carlo formulation for calculating the probability density function of a single scalar in a turbulent flow. Flow Turbulence and Combustion, 60:157-172, 1998.

M. Warshaw. Cloud droplet coalescence: statistical foundations and a onedimensional sedimentation model. Journal of the Atmospheric Sciences, 24: 278-286, 1967.

J. Wu and S. Menon. Aerosol dynamics in the near field of engine exhaust plumes. Journal of Applied Meteorology, 40:795-809, 2001. 


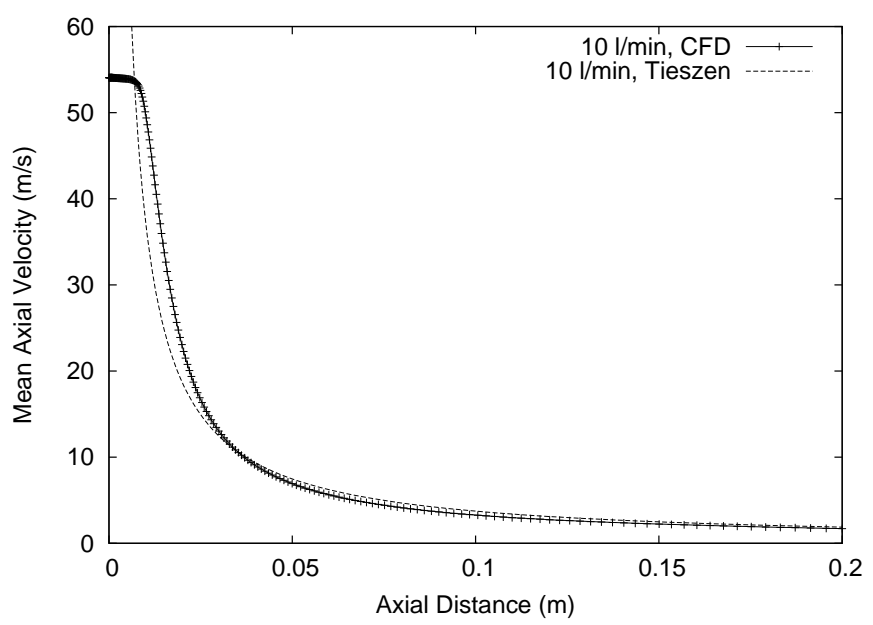

Fig. 1. Mean axial velocity along the jet axis. CFD solution compared with an empirical expression (Tieszen et al., 1996).

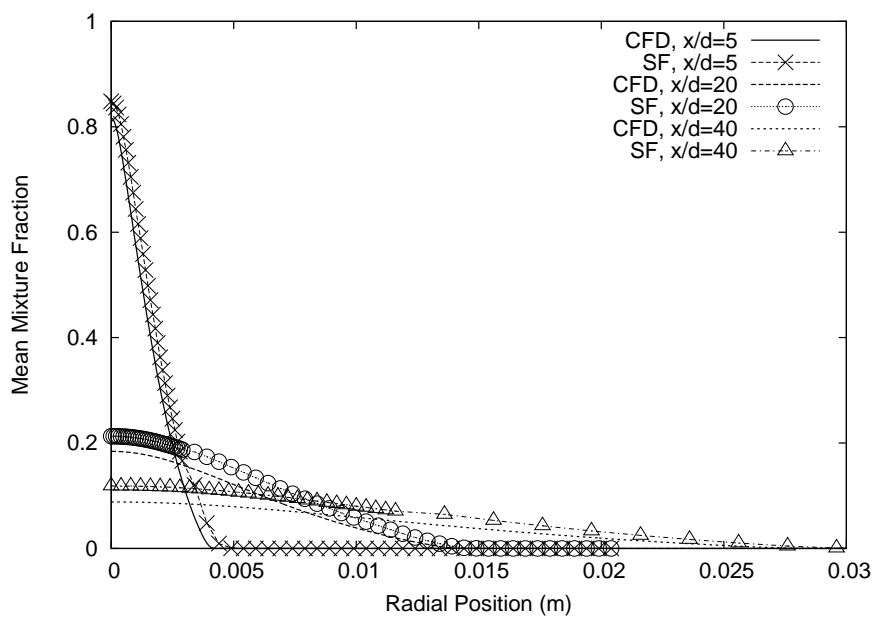

Fig. 2. Radial profile of mean mixture fraction at $x_{1} / d=5 \& 20$ downstream of the jet. The CFD solution and the time-marched SF solution are compared. 


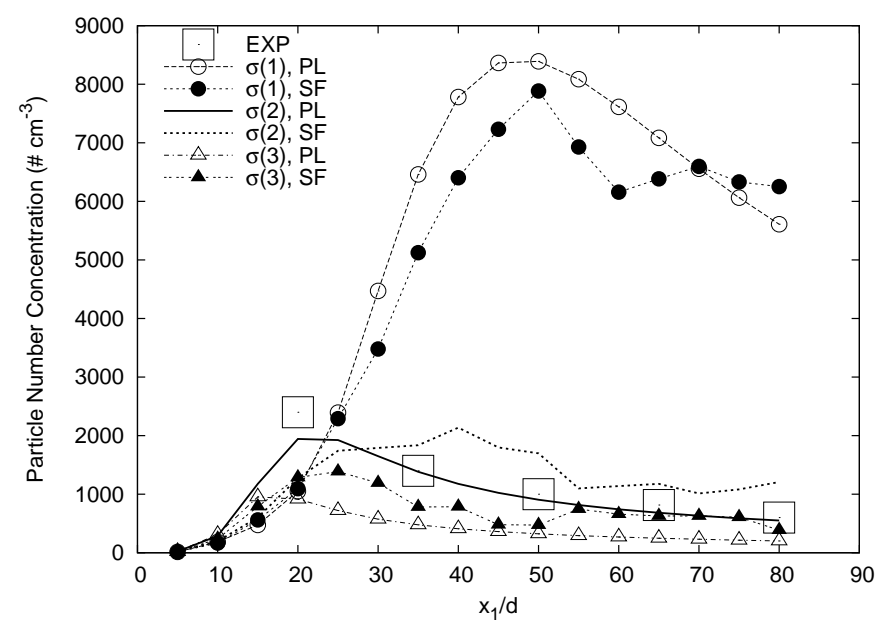

Fig. 3. Axial evolution of particle number concentration along centre-line. Calculations using different expressions for surface tension $\sigma(1), \sigma(2)$ and $\sigma(3)$ (Eqs. (28), (29) \& (30)). Calculations using SF (with $T_{e d d y}=k / \epsilon$ ) and plain, non-stochastic, method (PL) for each expression. Initial jet conditions: peak velocity $51.5 \mathrm{~m}$ $\mathrm{s}^{-1}$, temperature $413 \mathrm{~K}$, vapour concentration $360 \mathrm{ppm}$. Experimental data from Lesniewski (1997).

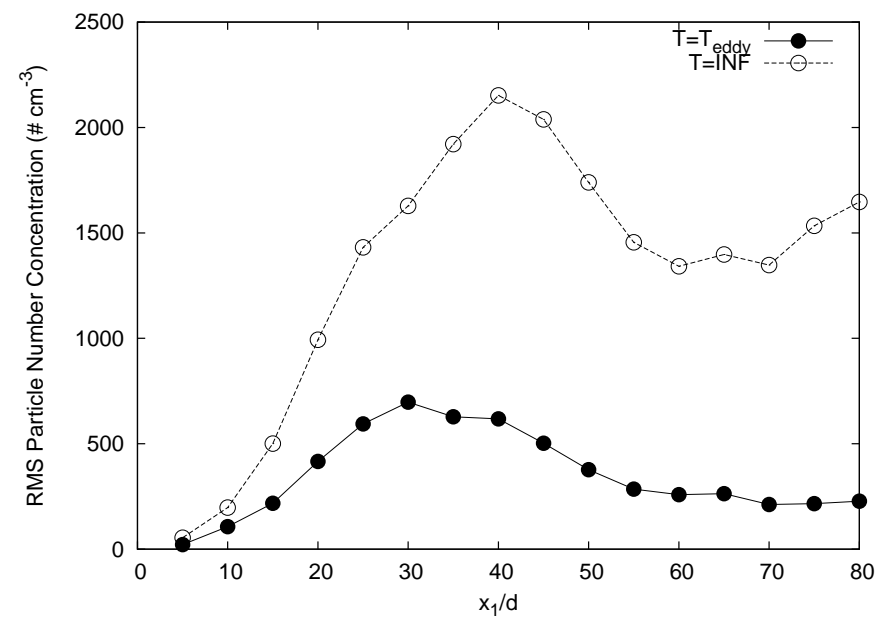

Fig. 4. RMS of particle number concentration along the jet centre-line predicted by Stochastic Fields method using both finite and infinite mixing timescale. Initial jet conditions: peak velocity $51.5 \mathrm{~m} \mathrm{~s}^{-1}$, temperature $413 \mathrm{~K}$, vapour concentration 360 ppm. 


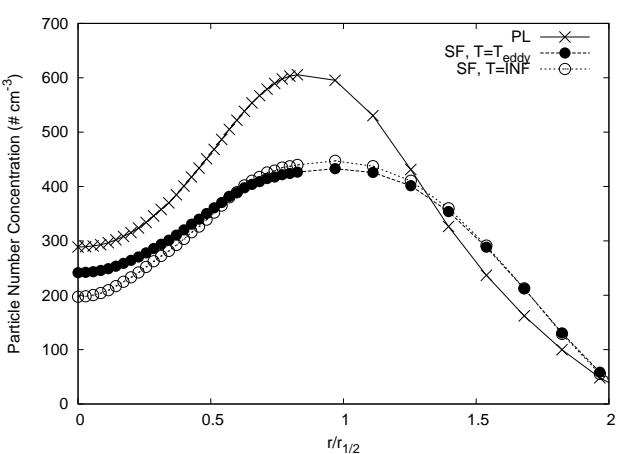

(a)

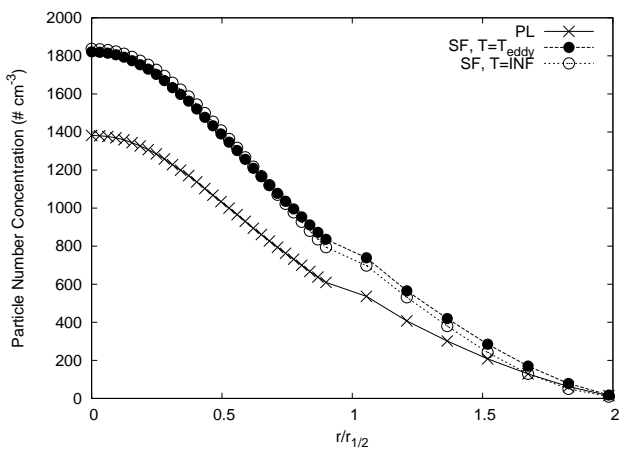

(c)

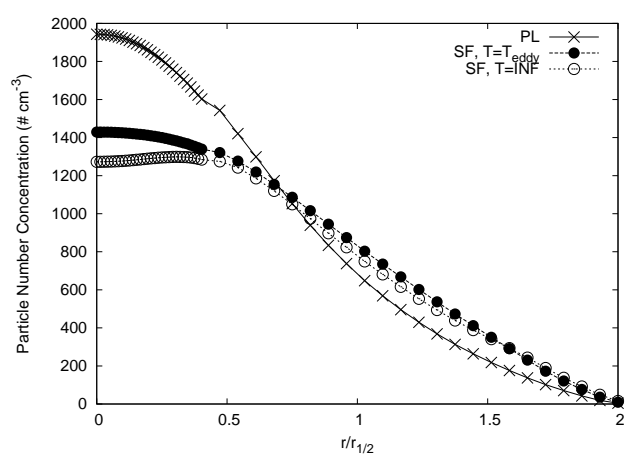

(b)

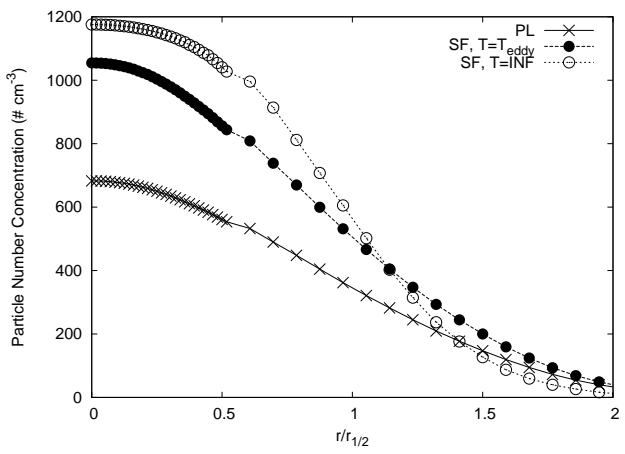

(d)

Fig. 5. Radial profiles of mean particle number density at $x_{1} / d=$ (a) 10 , (b) 20, (c) 35 and (d) 65. Results from plain non-stochastic solution and from Stochastic Fields with both finite and infinite mixing timescale. Initial jet conditions: peak velocity $51.5 \mathrm{~m} \mathrm{~s}^{-1}$, temperature $413 \mathrm{~K}$, vapour concentration $360 \mathrm{ppm}$. 


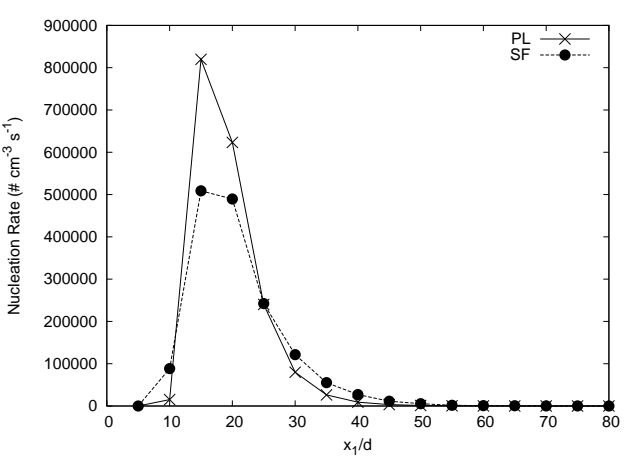

(a)

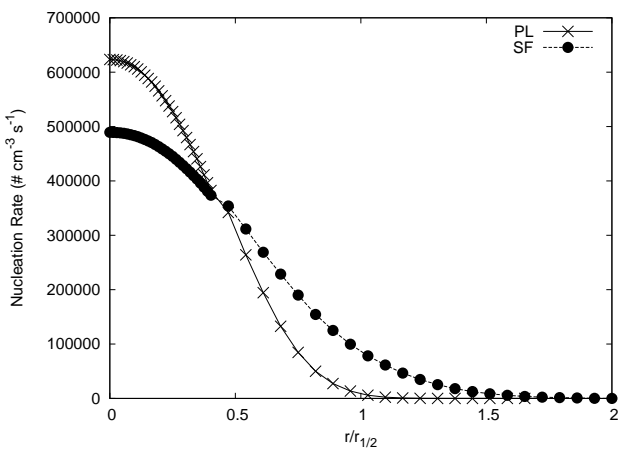

(c)

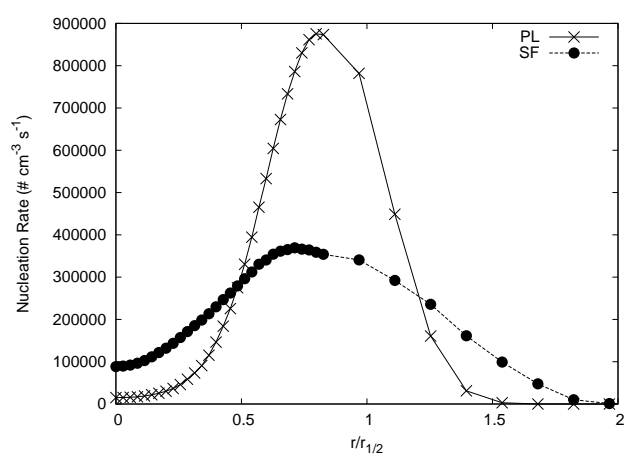

(b)

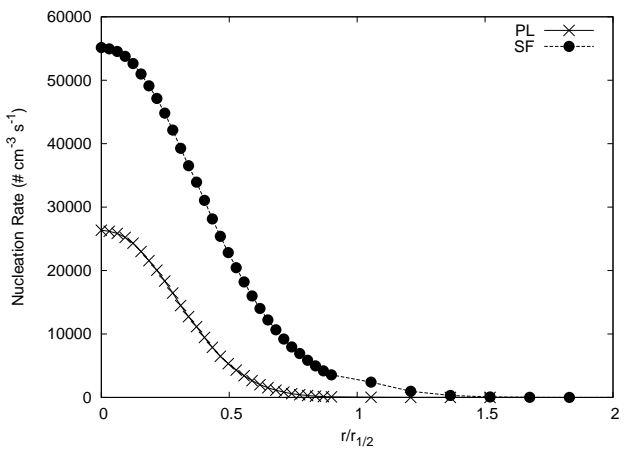

(d)

Fig. 6. Profiles of mean nucleation rate, (a) along the jet centre-line and radially at $x_{1} / d=$ (b) 10 , (c) 20 and (d) 35. Results from plain non-stochastic solution and from Stochastic Fields. Initial jet conditions: peak velocity $51.5 \mathrm{~m} \mathrm{~s}^{-1}$, temperature $413 \mathrm{~K}$, vapour concentration $360 \mathrm{ppm}$. 


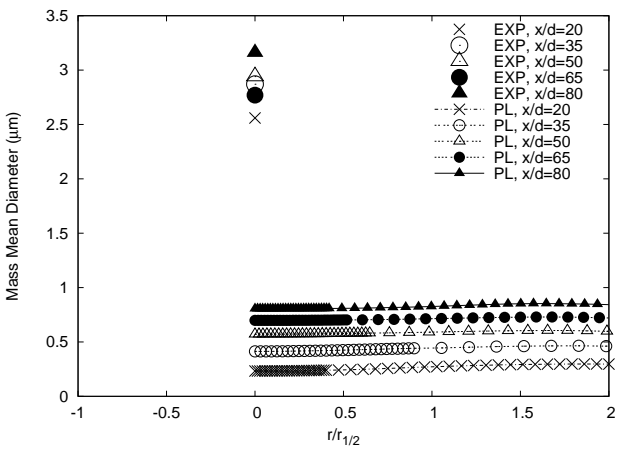

(a)

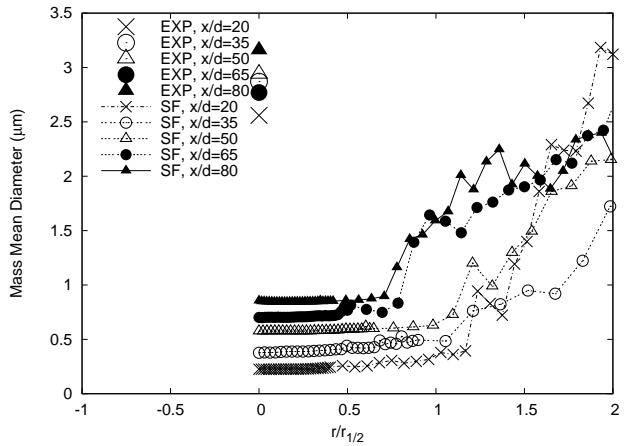

(c)

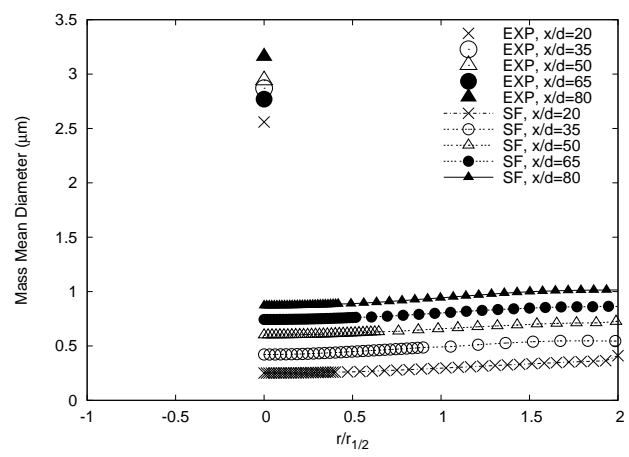

(b)

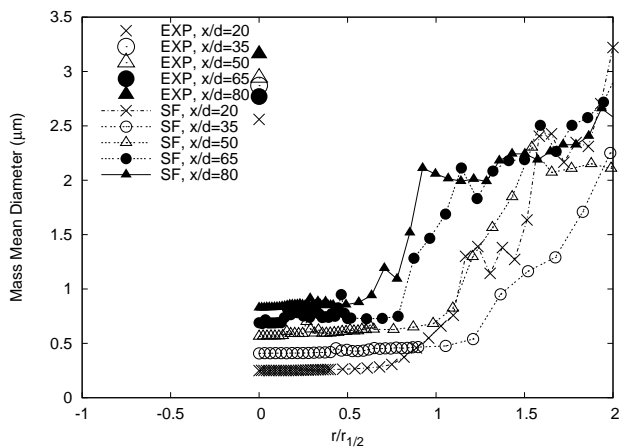

(d)

Fig. 7. Radial profiles of mass mean diameter. Calculated results from (a) plain non-stochastic solution; (b) Stochastic Fields with finite mixing timescale; (c) Stochastic Fields and infinite mixing timescale and (d) Stochastic Fields and infinite mixing timescale using an assumed monodisperse distribution. Initial jet conditions: peak velocity $51.5 \mathrm{~m} \mathrm{~s}^{-1}$, temperature $413 \mathrm{~K}$, vapour concentration 360 ppm. Experimental data from Lesniewski (1997). 


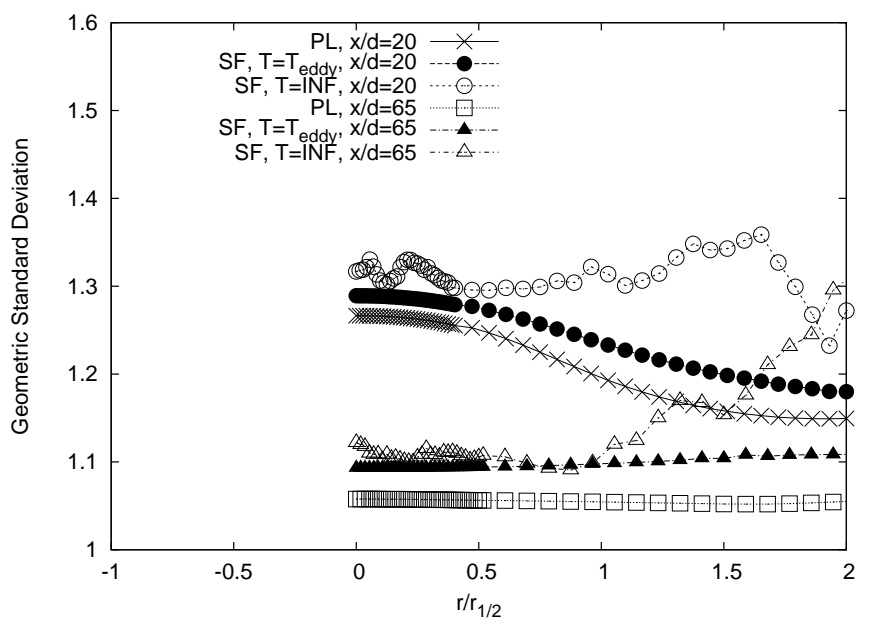

Fig. 8. Radial profiles of log-normal geometric standard deviation calculated using plain non-stochastic solution, Stochastic Fields with finite mixing timescale and Stochastic Fields with infinite mixing timescale.

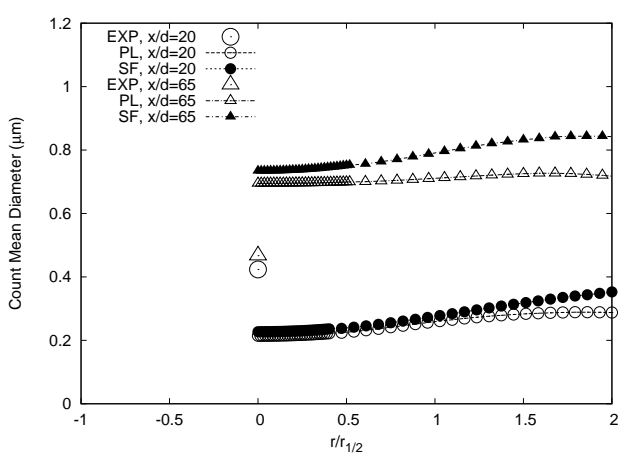

(a)

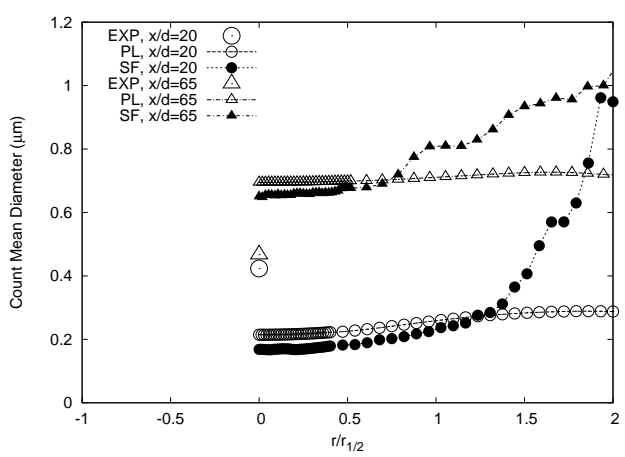

(b)

Fig. 9. Radial profiles of count mean diameter. Calculated results comparing (a) plain non-stochastic solution with Stochastic Fields with finite mixing timescale and (b) plain non-stochastic solution with Stochastic Fields with infinite mixing timescale. Initial jet conditions: peak velocity $51.5 \mathrm{~m} \mathrm{~s}^{-1}$, temperature $413 \mathrm{~K}$, vapour concentration 360 ppm. Experimental data from Lesniewski (1997). 


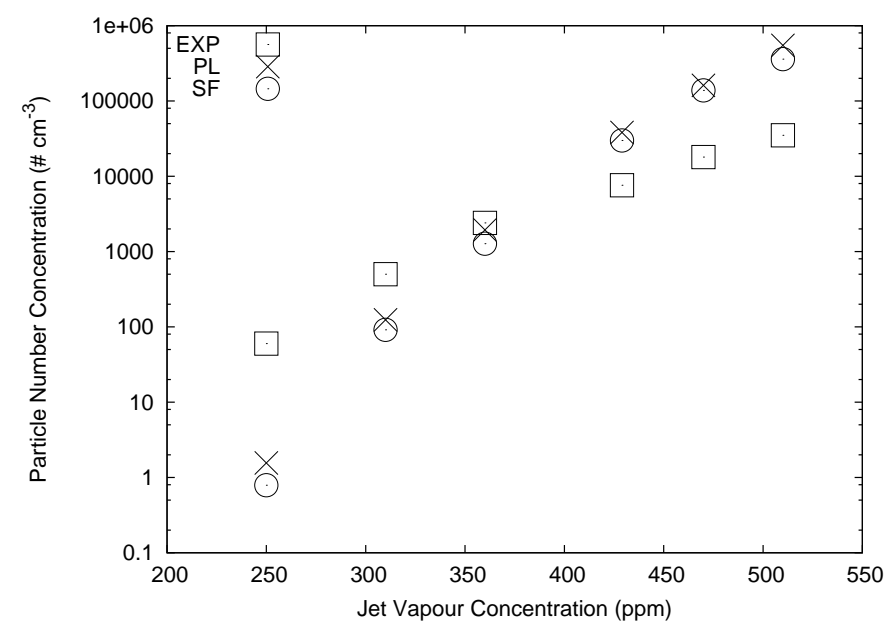

Fig. 10. Variation of particle number concentration on centre-line at $x_{1} / d=20$. Predictions using plain non-stochastic solution and Stochastic Fields. Experimental data from Lesniewski (1997).

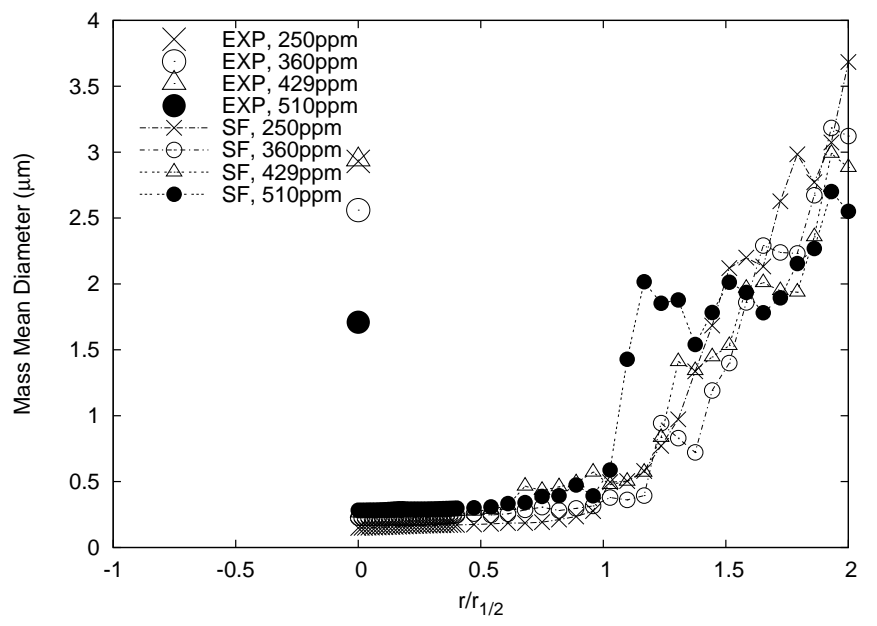

Fig. 11. Radial profiles of mass mean diameter at $x_{1} / d=20$ for different initial jet vapour concentrations. Predictions using plain non-stochastic solution and Stochastic Fields with infinite mixing timescale. Experimental data from Lesniewski (1997). 


\section{Table 1}

Properties and constants used in Dibutyl Phthalate aerosol process calculations. Data from Lesniewski and Friedlander (1998); Friedlander (2000); Pratsinis (1988) and Jacobson (1999).

\begin{tabular}{|l|l|}
\hline DBP molecular weight $(\mathrm{g}$ molecule & -1 \\
Saturation vapour pressure $(\mathrm{mm} \mathrm{Hg})$ & $m=278.4 / N_{A}$ \\
Surface tension $\left(\mathrm{g} \mathrm{s}^{-2}\right)$ & $\log P_{\text {sat }}=7.065-1666 / T-547700 / T^{2}$ \\
Condensed density $\left(\mathrm{g} \mathrm{cm}^{-3}\right)$ & $\rho_{p}=1.063-0.000826(T-273)$ \\
Molecular volume $\left(\mathrm{cm}^{3}\right)$ & $v_{m}=m / \rho_{p}$ \\
Mean free path $\left(\mathrm{cm}^{2}\right)$ & $\lambda=8.038 / \rho_{a} \times 10^{-6}$ \\
Gas viscosity $\left(\mathrm{g} \mathrm{cm}^{-1} \mathrm{~s}^{-1}\right)$ & $\mu=1.8 \times 10^{-4}$ \\
\hline
\end{tabular}

